\title{
A Novel Long Distance Fiber Bragg Grating Sensor System with Low Threshold Pump Power and High OSNR
}

\author{
Ronghua Chi' ${ }^{1}, 2$, Hangtao Zhao, Liya Li \\ ${ }^{1}$ School of Internet of things and software technology, Wuxi vocational college of science and \\ technology, Wuxi 214068, China \\ ${ }^{2}$ SoutheastUniversity,National Research Center for Optical SensingCommunications Integrated $\mathrm{Ne}$ \\ tworking, Nanjing 210096, China
}

\begin{abstract}
We propose a novel fiber Bragg grating (FBG) sensor system with low pump power and high optical signal to noise ratio (OSNR). This sensor system is based on a distributed Raman fiber amplifier and an Erbium doped fiber laser (EDFL). The EDFL cavity is composed by an FBG and a fiber loop mirror which decrease the laser threshold obviously. By recycling the residual Raman pump power as the EDFL pump, the overall system configuration was significantly simplified without any additional light source. The experiment results show that only $140 \mathrm{~mW}$ of $1470 \mathrm{~nm}$ Raman pump power can provide a stable output power with an optical signal-to-noise ratio over $50 \mathrm{~dB}$ even if the FBG is located at a $50 \mathrm{~km}$ remote sensing position.
\end{abstract}

Keywords: Long distance FBG sensor system, Raman fiber amplifier, Erbium dope fiber laser, OSNR.

\section{Introduction}

The Fiber Bragg grating (FBG) technology has attracted much interest in the field of optical sensors since the sensor based on FBG can provide the most simple ways to monitor a variety of external perturbations for example, temperature, strain, and pressure due to its high sensitivity, electro-magnetic immunity, compactness, and ease of fabrication [1-4]. A number of practical FBG based sensor systems have been implemented and demonstrated. In such FBG based sensing systems, one practical issue is to increase the transmission distance because their maximum transmission distance with a broadband light source is limited to $25 \mathrm{~km}$ mainly due to Rayleigh scattering induced optical noise as well as background signal loss with the transmission fiber. In order to increase the transmission distance, several novel methods based on Raman amplification have been suggested. For example, Y. Nakajima et al. proposed a novel method of the use of distributed Raman amplification in the signal transmission fiber to increase the sensing signal transmission distance over $50 \mathrm{~km}$ in a passive FBG sensor system [5]. However, the requirement of two separate light sources of a broadband light source and a Raman pump could be a limiting factor due to its higher cost. P.-C. Peng et al. proposed an advanced method of the use of the linear cavity Raman laser configuration based on a FBG and fiber loop mirror for a long-distance strain sensing system [6]. Although the use of Raman laser configuration eliminates the requirement of an additional broadband light source and improves the sensing signal quality, but about more than $250 \mathrm{~mW}$ threshold pump power is often need this will take higher system cost.

In this paper, we demonstrate a novel and simple, Raman amplifier plus Erbium doped fiber laser (EDFL)-based long-distance sensing system. Our proposed sensing system has only one pump source for distributed Raman amplification and EDFL in the transmission fiber without any additional broadband light source and the residual pump power after the transmission fiber is recycled for the erbium-doped fiber. Using the proposed scheme, we obtain a remote sensing operation of temperature measurement at a location of $50 \mathrm{~km}$ and deliver the sensing signals through the transmission fiber with distributed Raman amplification. High quality of sensing signals with a $\sim 50 \mathrm{~dB}$ OSNR is readily achieved even after the $50 \mathrm{~km}$ transmission when the Raman pump power is $140 \mathrm{~mW}$. 


\section{Experimental Setup}

Fig. 1 shows the experimental setup for the proposed long-distance remote sensing system. A 1470$\mathrm{nm}$ Raman pump with rated power of $250 \mathrm{~mW}$ is chosen due to its high absorption. The pump power could be launched into a $50 \mathrm{~km}$ long standard single mode fiber (SMF) with $\sim 0.2 \mathrm{~dB} / \mathrm{km}$ attenuation via a $1550 / 1470 \mathrm{~nm}$ WDM coupler. Then, the residual pump power after the SMF was launched into a $7 \mathrm{~m}$ long EDF via two 1550/1470 nm WDM couplers. The residual Raman pump was reused as a pump source for pumping the $7 \mathrm{~m}$ EDF. The peak absorption coefficient of the EDF at $1530 \mathrm{~nm}$ was $6 \mathrm{~dB} / \mathrm{m}$. A fiber loop mirror acting as one of the cavity mirror with a wide band reflective spectrum and high reflectivity. The fiber loop mirror is composed by a $3 \mathrm{~dB}$ coupler. The other cavity mirror is a FBG. We used the UV beam scanning method with a phase mask to fabricate the FBG in boron (B)-Germanium (Ge) codoped silica based photosensitive fiber. The center wavelength of the FBG with a $0.2 \mathrm{~nm}$ spectral width was $1550.2 \mathrm{~nm}$ and its measured reflectivity was $\sim 85 \%$.

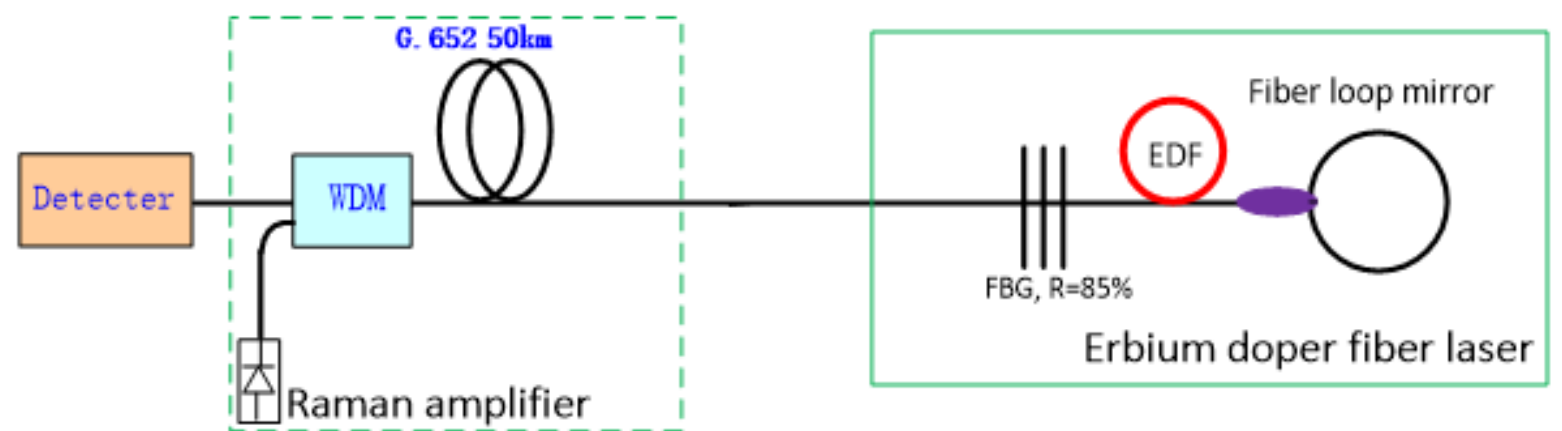

Fig.1 Experimental setup for Raman amplifier plus EDFL-based long-distance remote sensor system

\section{Experiment Results and Discussion}

The FBG was placed into a temperature heating oven for temperature sensitivity measurement. The temperature-heating oven used in this experiment has a temperature tuning range between $-40{ }^{\circ} \mathrm{C}$ and $100{ }^{\circ} \mathrm{C}$ and good temperature stability. Resolution of the sensor system was detected by the OSA with resolution of $0.01 \mathrm{~nm}$. Fig. 2 shows the spectrum of the OSA detected. Curve (a) is the detected signal when the Raman pump power is $75 \mathrm{~mW}$ and the residual pump power after the transmission fiber (EDFL pump power) is $5.25 \mathrm{~mW}$. We can see there is only ASE source. When the Raman pump power is increased the residual pump power is increased. When the residual Raman pump power is equal to the EDFL threshold (about 5.42 $\mathrm{mW}$ ), the EDFL begin to lase which showed in curve (b). Curve (c) show the laser signal when the pump power is $85 \mathrm{~mW}$ and the residual power is $5.7 \mathrm{~mW}$. But we can see the OSNR is still very small. When the Raman pump power is reached $140 \mathrm{~mW}$ and the residual EDFL pump power is $10.9 \mathrm{~mW}$, the detected signal OSNR is nearly 50dB, which was shown in Fig.3.

Fig. 4 shows the detected laser signal spectrum stability at room temperature. The spectrum is swept every one minute. We can see that the signal is very stable. The signal peak is kept at $1550.178 \mathrm{~nm}$. The fluctuation of the output signal peak power less than $0.2 \mathrm{~dB}$ can be obtained during 16 minutes sweeping time. 


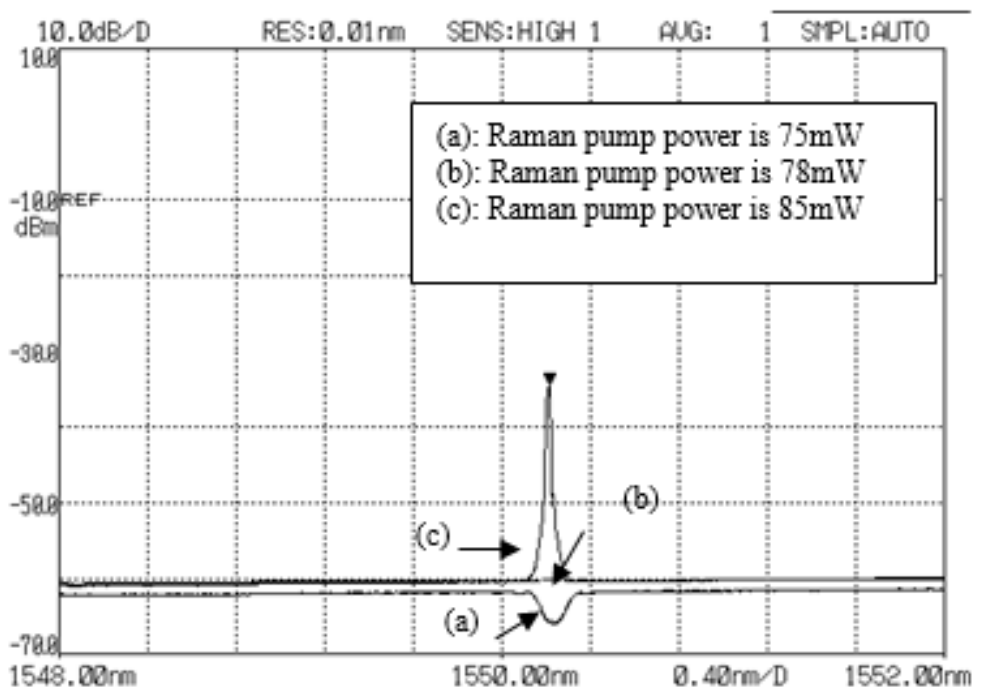

Fig.2 Detected signal power around threshold.

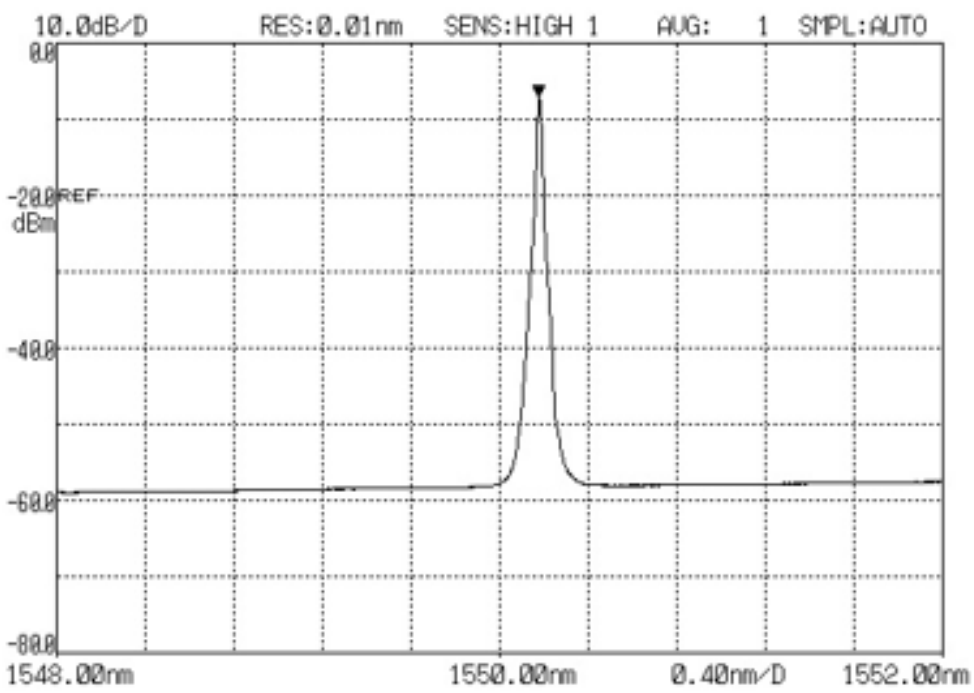

Fig.3 Laser spectrum when the Raman pump power is $140 \mathrm{~mW}$ at room temperature $(\mathrm{OSNR}=50 \mathrm{~dB})$

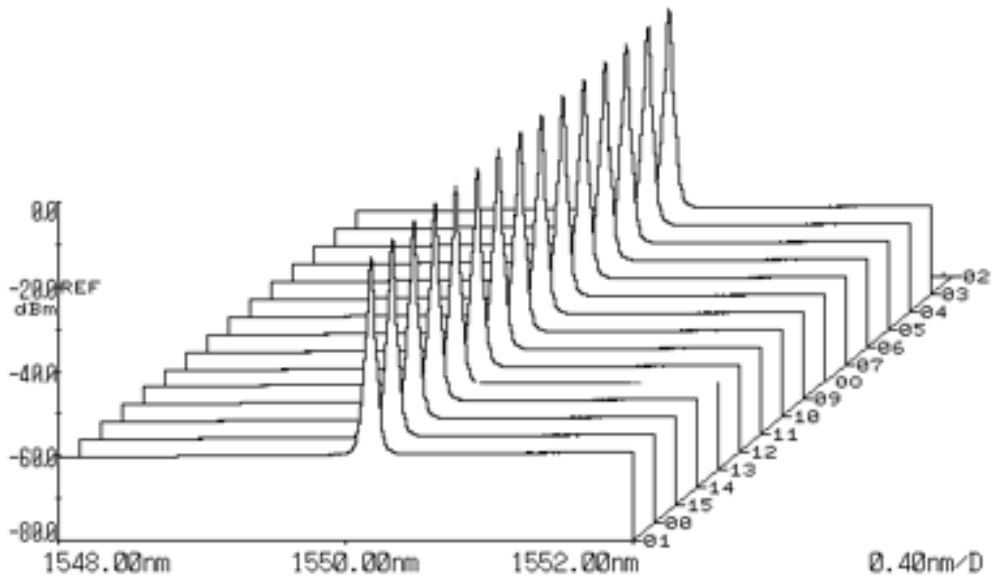

Fig.4 The output laser signal spectrum stability (16minutes) 


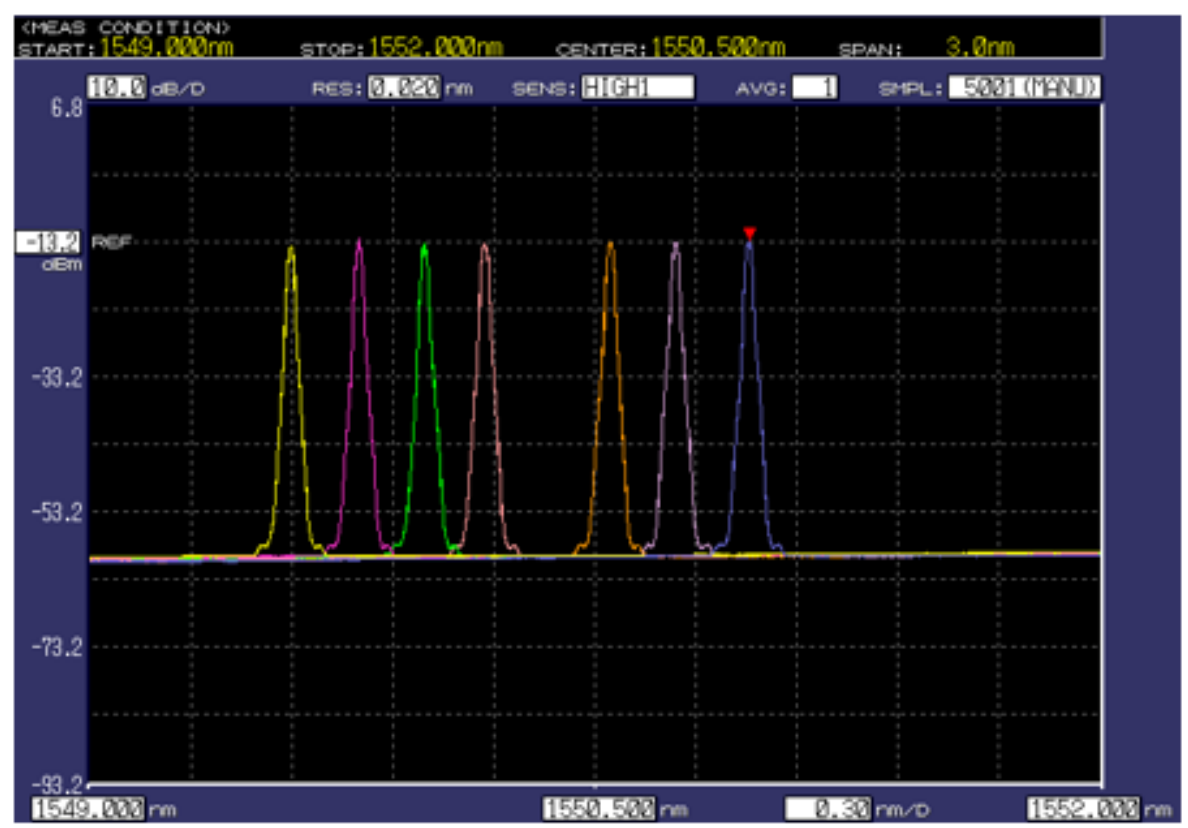

Fig. 5 Laser wavelength shifts from $-40{ }^{\circ} \mathrm{C}$ to $100{ }^{\circ} \mathrm{C}$

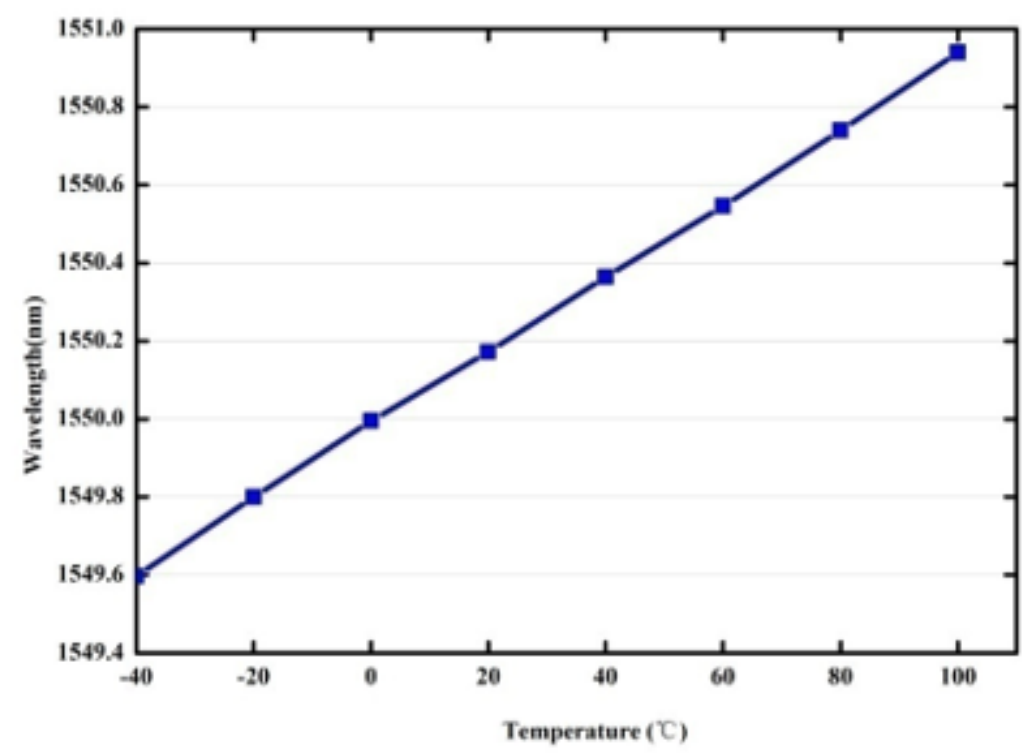

Fig.6 Laser centre wavelength as a function of Temperature

Fig. 5 shows the wavelength shifts of FBG when the oven temperature is changed from $-40{ }^{\circ} \mathrm{C}$ to $100{ }^{\circ} \mathrm{C}$. We can see that the signal wavelength shift from $1549.6 \mathrm{~nm}$ to $1550.94 \mathrm{~nm}$. And the signal has high OSNR over $45 \mathrm{~dB}$. And the output power is very stable.

Fig. 6 illustrates the wavelength as a function of the temperature. The FBG temperature is from $40^{\circ} \mathrm{C}$ to $100^{\circ} \mathrm{C}$, the wavelength is changed from $1549.6 \mathrm{~nm}$ to $1550.94 \mathrm{~nm}$. The laser wavelength and FBG temperature shows a good linear relationship. And the temperature sensitivity is $9.571 \mathrm{pm}$ $/{ }^{\circ} \mathrm{C}$.Our experiment has shown that our system has good repeatability.

We also investigate the relationship between signal peak power and Raman pump power. The result is shown in Fig.7. We can see that the signal peak power is increased with Raman pump power. When the Raman pump power is increased from $80 \mathrm{~mW}$ to $250 \mathrm{~mW}$, the signal power is increased from very small power to $0.15 \mathrm{~mW}$. At the same time, the signal SNR value increased rapidly with Raman pump power when the pump power increased from 80 to $100 \mathrm{~mW}$. When pump is $100 \mathrm{~mW}$, the SNR is already nearly $40 \mathrm{~dB}$ high. But after $100 \mathrm{~mW}$, SNR increased very slowly. 


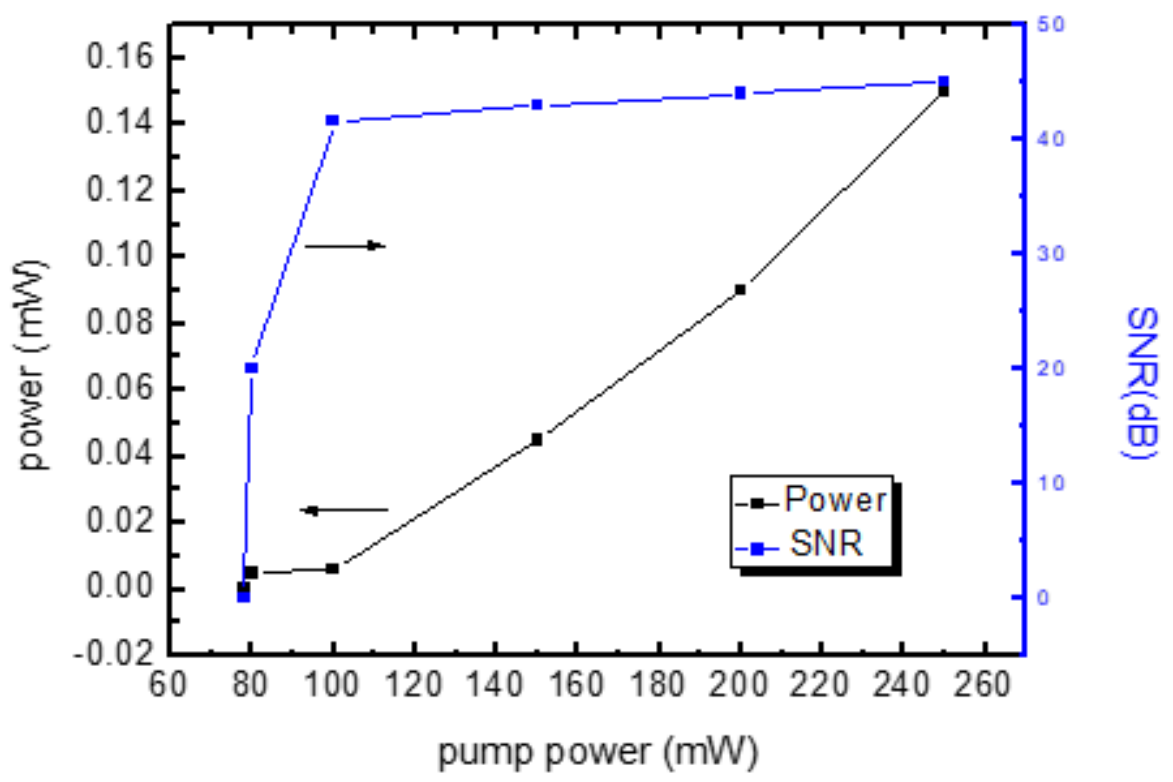

Fig.7 Detected signal peak power/SNR VS.Raman pump power

\section{Summary}

A long distance FBG sensor system using Raman amplifier and a linear-cavity Erbium doped fiber laser scheme has been proposed. Our proposed sensing system has only one pump source for distributed Raman amplification and EDFL in the transmission fiber without any additional broadband light source and the residual pump power after the transmission fiber is recycled for the erbium-doped fiber. Experiment results show a detected signal with stable and intense peak power even if the FBG was located at a 50-km far sensing position. With the help of fiber loop mirror, the EDFL laser has very small threshold $5.42 \mathrm{~mW}$ corresponding to $78 \mathrm{~mW}$ Raman pump power. Also high optical SNR of $50 \mathrm{~dB}$ is obtained with only $140 \mathrm{~mW}$ Raman pump power and corresponding 10.9 $\mathrm{mW}$ residual power for EDFL. The proposed FBG sensor system can be used for long-haul structure.

\section{References}

[1]. Jia Shi, Yuye Wang, Degang Xu et al.,Temperature Sensor Based on Fiber Ring Laser With Sagnac Loop, IEEE PHOTONICS TECHNOLOGY LETTERS, 28( 7), APRIL 1, 2016.

[2]. Montserrat Fernandez-Vallejo, Mikel Bravo, and Manuel Lopez-Amo, Ultra-Long Laser Systems for Remote Fiber Bragg Gratings Arrays Interrogation,IEEE PHOTONICS TECHNOLOGY LETTERS, VOL. 25, NO. 14, JULY 15, 2013.

[3]. Daniel Leandro, Ver'onica deMiguel-Soto, and Manuel L'opez -Amo, High-Resolution Sensor System Using a Random Distributed Feedback Fiber Laser,JOURNAL OF LIGHTWAVE TECHNOLOGY, VOL. 34, NO. 19, OCTOBER 2016.

[4]. Junhao Hu, Zhihao Chen, and Changyuan Yu, "IEEE150-km Long Distance FBG Temperature and Vibration Sensor System Based on Stimulated Raman Amplification," Journal of lightwave technology, Vol. 30(2012), No. 8, April 15.

[5]. Y. Nakajima, Y. Shindo, and T. Yoshikawa, "Novel concept as long-distance transmission FBG sensor system using distributed Raman amplification," in Proc. 16th International Conference on Optical FiberSensors (Nara Japan, October 2003), Th1-4.

[6]. P.-C. Peng, H.-Y Tseng, and Sien Chi, "Long-distance FBG sensor system using a linear-cavity fiber Raman laser scheme,” IEEE Photon. Technol. Lett. 16 (2004), 575-577. 